\title{
Hydrodynamic Drag on a Compact Star Orbiting a Supermassive Black Hole
}

\section{Citation}

Narayan, Ramesh. 2000. "Hydrodynamic Drag on a Compact Star Orbiting a Supermassive Black Hole." The Astrophysical Journal 536 (2): 663-67. https://doi.org/10.1086/308956.

\section{Permanent link}

http://nrs.harvard.edu/urn-3:HUL.InstRepos:41384926

\section{Terms of Use}

This article was downloaded from Harvard University's DASH repository, and is made available under the terms and conditions applicable to Other Posted Material, as set forth at http:// nrs.harvard.edu/urn-3:HUL.InstRepos:dash.current.terms-of-use\#LAA

\section{Share Your Story}

The Harvard community has made this article openly available.

Please share how this access benefits you. Submit a story.

\section{Accessibility}




\title{
Hydrodynamic Drag on a Compact Star Orbiting a Supermassive Black Hole
}

\author{
Ramesh Narayan \\ Harvard-Smithsonian Center for Astrophysics, 60 Garden St., Cambridge, MA 02138
}

\begin{abstract}
The proposed Laser Interferometer Space Antenna is expected to detect gravitational waves from neutron stars and stellar-mass black holes spiraling into supermassive black holes in distant galactic nuclei. Analysis of the inspiral events will require careful comparison of the observed signals with theoretical waveform templates. The comparison could be seriously compromised if non-gravitational torques modify the orbit of the star. This paper estimates the torque exerted on an orbiting star as a result of hydrodynamic interactions with an accretion flow around the supermassive black hole. It is argued that the majority of inspiral events will take place in low luminosity galactic nuclei in which the mass accretion rate is low and the accretion occurs via an advection-dominated flow. The hydrodynamic torque is negligibly small in such systems and will have no effect on gravitational wave experiments.
\end{abstract}

Subject headings: Accretion, accretion disks — black hole physics — gravitation — relativity galaxies: nuclei - gravitational waves

\section{Introduction}

When a compact object spirals into a much more massive object via the emission of gravitational radiation, the waves carry information on the multipoles of the central mass (Ryan 1995). Therefore, by monitoring the gravitational wave signal one could explore the spacetime geometry outside the central body, as anticipated by Abramovici et al. (1992). If the object is a black hole, it should be possible to verify its black hole nature and measure its spin parameter $(a / M)$ from the ratios of the multipoles.

Practical application of this idea is expected to become feasible in the next decade or two. When a neutron star or a stellar-mass black hole spirals into a supermassive black hole (SMBH) in a galactic nucleus, the gravitational waves have periods in the range $10-10^{4} \mathrm{~s}$. Such periods are well-suited for detection with the proposed Laser Interferometer Space Antenna (LISA, see Danzmann et al. 1996, Bender et al. 1996, Folkner 1998, Cutler 1998). LISA could detect the inspiral of a $10 M_{\odot}$ black hole into a $10^{6} M_{\odot} \mathrm{SMBH}$ out to a redshift $\sim 1$. At these distances event rates of up to several per year are likely (Hils \& Bender 1995, Sigurdsson 1997).

Both the detection and the analysis of the signals will require a very clean system with no significant perturbation of the orbiting star. Is this likely? In an interesting series of papers, Chakrabarti (1993, 1996) and Molteni, Gerardi \& Chakrabarti (1994) showed that, under some cirumstances, the hydrodynamic interaction between the orbiting star and an accretion disk surrounding the SMBH could be so strong that the inspiral may be halted altogether and the star may be trapped in a stable orbit. Chakrabarti's scenario is an extreme one that involves a very strong hydrodynamic interaction. It presumably occurs only rarely. However, even if the interaction is orders of magnitude weaker than the level required by Chakrabarti, it

\footnotetext{
${ }^{1}$ rnarayan@cfa.harvard.edu
} 
could still pose a serious problem. The detection of inspiral events involves matching the observed signal with a theoretical template covering many hundreds or thousands of wave periods, and even a tiny perturbation could be fatal.

Motivated by this, we investigate the following simple question: What is the expected strength of the hydrodynamic interaction in a "typical" galactic nucleus, and how significant is the orbital perturbation due to this interaction?

Before embarking on a quantitative analysis, we make two points.

First, only a small fraction of galaxies harbor active galactic nuclei. Active nuclei, such as quasars, Seyferts, etc. (see Krolik 1999 for a review), are thought to consist of SMBHs accreting mass at rates close to or exceeding the Eddington rate $\dot{M}_{\text {Edd }}$. The accretion is believed to proceed via a thin accretion disk (Shakura \& Sunyaev 1973, Novikov \& Thorne 1973) when $\dot{M} \lesssim \dot{M}_{\text {Edd }}$, or a slim accretion disk (Abramowicz et al. 1988) for $\dot{M} \gtrsim \dot{M}_{\text {Edd }}$. Chakrabarti's work is focused on such high- $\dot{M}$ SMBHs; indeed, he explicitly considers super-Eddington accretion in his analysis. However, the vast majority of galactic nuclei are much dimmer than typical quasars or Seyferts. It has become clear in recent years that these "normal" nuclei also contain SMBHs (e.g. Magorrian et al. 1998), but that the accretion rates are much below $\dot{M}_{\text {Edd }}$, perhaps as low as $\dot{M} \sim 10^{-2}-10^{-4} \dot{M}_{\text {Edd }}$. The lower $\dot{M}$ implies that there should be less gas in the vicinity of the $\mathrm{SMBH}$ and therefore a weaker frictional drag on an orbiting star.

Second, at the low values of $\dot{M}$ relevant for normal galactic nuclei, there is increasing evidence that the gas flows have a different character from the flows found in bright nuclei; the accretion does not occur in a thin or slim disk but via an advection-dominated accretion flow (ADAF, see Narayan \& Yi 1994, 1995a,b, Abramowicz et al. 1995, Ichimaru 1977, Rees et al. 1982; also Kato, Fukue \& Mineshige 1998 and Narayan, Mahadevan \& Quataert 1998 for reviews). For a given value of $\dot{M}$, the gas density in an ADAF is significantly lower than that in a thin disk. This further reduces the hydrodynamic drag on an orbiting star.

In $\S 2$ of this paper we estimate the hydrodynamic torque on a compact star orbiting inside an ADAF, and in $\S 3$ we compare this with the torque that results from gravitational wave emission. We conclude with a discussion in $\S 4$.

\section{Hydrodynamic Drag on a Compact Star Orbiting Inside an ADAF}

Consider a compact star of mass $M_{c}=10 m_{1} M_{\odot}$ orbiting a SMBH of mass $M_{\mathrm{SMBH}}=10^{6} m_{6} M_{\odot}$. For simplicity, we assume that the star is in a circular orbit of radius $R=r R_{g}$, where $R_{g}=G M_{\mathrm{SMBH}} / c^{2}$. We also work within a Newtonian framework. This is adequate in view of the various other approximations we make and considering that we seek only order of magnitude estimates. The orbital velocity of the star is given by the Keplerian formula,

$$
v_{K}=\frac{c}{r^{1 / 2}}
$$

Let the mass accretion rate onto the SMBH be $\dot{M}_{\mathrm{SMBH}}$, and let us express this in Eddington units:

$$
\begin{gathered}
\dot{M}_{\mathrm{SMBH}}=\dot{m} \dot{M}_{\mathrm{Edd}}, \\
\dot{M}_{\mathrm{Edd}}=\frac{4 \pi G M_{s} m_{p}}{\eta \sigma_{T} c}=1.4 \times 10^{24} m_{6} \mathrm{~g} \mathrm{~s}^{-1},
\end{gathered}
$$

where $m_{p}$ is the proton mass and $\sigma_{T}$ is the Thomson cross-section of the electron. The parameter $\eta$ refers to the radiative efficiency of the accretion assumed for the purpose of defining the Eddington rate; we use 
$\eta=0.1$ for calculating the numerical value given in the right hand side of equation (3). Note that the choice of $\eta$ in the definition of $\dot{M}_{\text {Edd }}$ is purely conventional. The actual radiative efficiency in any particular accretion flow could be different from 0.1 .

Let us define also the Salpeter time, which is the mass $e$-folding time of an object that accretes at the Eddington rate:

$$
t_{S}=\frac{M_{\mathrm{SMBH}}}{\dot{M}_{\mathrm{Edd}}}=\frac{\eta \sigma_{T} c}{4 \pi G m_{p}}=4.5 \times 10^{7} \mathrm{yr} .
$$

Once again, we have set $\eta=0.1$ on the right.

We assume that the accretion flow around the SMBH occurs via an ADAF. The key characteristic of an ADAF is that the thermal energy released as the gas falls into the potential well of the central mass is not radiated (as in a thin disk) but is retained in the gas and advected down to the center (Narayan \& Yi 1994). This causes a number of important effects on the dynamics of the gas.

First, since all the binding energy is retained as thermal energy, the gas becomes extremely hot and the temperature approaches the virial limit. Equivalently, the isothermal sound speed $c_{s} \equiv \sqrt{p / \rho}$, where $p$ is the pressure and $\rho$ is the density, approaches the Keplerian speed $v_{K}$. One consequence is that the gas does not take up a disk-like configuration (as in a thin accretion disk), but has a quasi-spherical morphology (Narayan \& Yi 1995a). Another consequence is that the orbital velocity of the gas is significantly sub-Keplerian; a typical value is $v_{\phi} \sim v_{K} / 3$. Both of these effects simplify our analysis considerably since they imply that the magnitude of the hydrodynamic drag is insensitive to the orientation of the stellar orbit relative to the angular momentum vector of the accreting gas. The radial velocity $v_{R}$ of the accreting gas is quite large, roughly $v_{R} \sim \alpha v_{K}$, where $\alpha$ is the standard dimensionless viscosity parameter (Shakura \& Sunyaev 1973). For an ADAF, $\alpha$ typically lies in the range 0.3 (Esin, McClintock \& Narayan 1997) to 0.1 (Quataert \& Narayan 1999). Thus the radial velocity of the gas is a substantial fraction of $v_{K}$.

We can now express the density $\rho$ of the accreting gas in terms of the mass accretion rate:

$$
\rho=\frac{\dot{M}_{\mathrm{SMBH}}}{4 \pi R^{2} v_{R}}=\frac{\dot{m}}{4 \pi \alpha} \frac{M_{\mathrm{SMBH}}}{t_{S}} \frac{c^{3}}{\left(G M_{\mathrm{SMBH}}\right)^{2}} \frac{1}{r^{3 / 2}},
$$

where we have expressed $\dot{M}_{\mathrm{SMBH}}$ in terms of the Eddington rate and set $v_{R}=\alpha v_{K}$.

Ostriker (1999) has estimated the drag force $F_{d f}$ on a mass $M_{c}$ moving through a uniform gas of density $\rho$ with relative velocity $v_{r e l}$ :

$$
F_{d f}=-4 \pi I\left(\frac{G M_{c}}{v_{r e l}}\right)^{2} \rho
$$

where the negative sign indicates that the force acts in the opposite direction to the velocity of the mass. The coefficient $I$ depends on the Mach number, $\mathcal{M} \equiv v_{\text {rel }} / c_{s}$, of the relative motion. For $\mathcal{M} \ll 1$, Ostriker finds $I \rightarrow \mathcal{M}^{3} / 3$, while for $\mathcal{M} \gg 1, I \rightarrow \ln \left(R_{\max } / R_{\min }\right)$, where $R_{\max }$ is the size of the gaseous system and $R_{\text {min }}$ is the effective size of the mass $M_{c}$.

In our problem, $v_{r e l} \sim c_{s} \sim v_{K}$ and the Mach number is of order unity. We could therefore use either the subsonic or supersonic estimate of $I$. We use the supersonic estimate as it gives a larger force and thereby provides an upper limit on the hydrodynamic drag. We set $R_{\max }$ equal to the size of the system, namely the local radius $R$. The choice of $R_{\min }$ is less obvious since the radius of the star, the natural choice, is inappropriate for a compact star. We set $R_{\text {min }}$ equal to the gravitational capture radius, $G M_{c} / v_{r e l}^{2}$, since Ostriker's linear analysis is valid only for gas streamlines with impact parameters larger than this radius. 
Setting $v_{r e l}=v_{K}$, we then find that

$$
I \sim \ln \left(\frac{R v_{r e l}^{2}}{G M_{c}}\right)=\ln \left(\frac{M_{\mathrm{SMBH}}}{M_{c}}\right)=12+\ln \left(\frac{m_{6}}{m_{1}}\right) .
$$

We use $I=10$ in numerical estimates.

Let us define the hydrodynamic drag time scale $t_{h d}$ to be the $e$-folding time for the specific angular momentum of the orbiting star. Thus

$$
t_{h d} \equiv \frac{M_{c} v_{K}}{\left|F_{d f}\right|}=\frac{c^{3}}{4 \pi I G \rho G M_{c}}\left(\frac{v_{r e l}}{v_{K}}\right)^{2} \frac{1}{r^{3 / 2}} .
$$

Setting $v_{r e l} \sim v_{K}$, and substituting for $\rho$ from equation (5), we find

$$
t_{h d}=\frac{\alpha}{I \dot{m}} \frac{M_{\mathrm{SMBH}}}{M_{c}} t_{S}=10^{5} \frac{\alpha}{I \dot{m}} \frac{m_{6}}{m_{1}} t_{S} .
$$

Putting in numerical values, this gives

$$
t_{h d} \sim 4.5 \times 10^{10} \frac{m_{6}}{\dot{m} m_{1}} \mathrm{yr},
$$

where we have used "standard" parameter values: $\alpha=0.1, I=10, \eta=0.1$.

ADAFs are present only for low values of $\dot{m} \lesssim \alpha^{2} \sim 10^{-1}-10^{-2}$ (Narayan \& Yi 1995b, Esin et al. 1997). The galactic nuclei we are interested in probably have even lower accretion rates: $10^{-4} \lesssim \dot{m} \lesssim 10^{-2}$. For such values of $\dot{m}$, we see that the hydrodynamic drag on an orbiting star is extremely small. In fact, two further effects make the drag even lower than the above estimate:

1. At radii $r \lesssim 10$, which is the region we are interested in for gravitational wave studies, the radial velocity of the ADAF is not $\alpha v_{K}$ but closer to $v_{K}$ (Narayan, Kato \& Honma 1997, Chen, Abramowicz \& Lasota 1997). This causes the density $\rho$ to decrease and the time scale $t_{h d}$ to increase by a factor $\sim 1 / \alpha \sim 10$.

2. Blandford \& Begelman (1999) suggested, following earlier work by Narayan \& Yi (1994, 1995a), that there could be significant mass outflows from ADAFs. As a result, close to the SMBH, $\dot{m}$ may be one or two

orders of magnitude lower than at large radii. This would again reduce the hydrodynamic drag on a star orbiting close to the SMBH.

\section{Comparison of Hydrodynamic and Gravitational Wave Torques}

The angular momentum of a binary system consisting of a SMBH and a compact star is given by $J=\mu \sqrt{G M a}$, where $M=M_{\mathrm{SMBH}}+M_{c} \approx M_{\mathrm{SMBH}}$ is the total mass, $\mu=M_{\mathrm{SMBH}} M_{c} / M \approx M_{c}$ is the reduced mass, and $a$ is the radius of the orbit. The angular frequency of the orbit is equal to $\sqrt{G M / a^{3}}$.

The time scale on which $J$ changes as a result of gravitational wave emission is (e.g. Shapiro \& Teukolsky 1983)

$$
t_{g w}=\frac{J}{|d J / d t|}=\frac{5}{32} \frac{c^{5}}{G^{3}} \frac{a^{4}}{M^{2} \mu} .
$$

Let us express this in practical units. We write $a=10 r_{1} R_{g}$, and define $P_{2}=P / 100 \mathrm{~s}$, where $P=\pi / \Omega$ is the period of the quadrupolar gravitational waves (this period is equal to one half the period of the orbit). 
Then

$$
t_{g w}=24 \frac{m_{6}^{2} r_{1}^{4}}{m_{1}} \mathrm{yr}=0.35 \frac{P_{2}^{8 / 3}}{m_{1} m_{6}^{2 / 3}} \mathrm{yr}
$$

The time to merger is given by

$$
t_{m}=\frac{t_{g w}}{8}=3.0 \frac{m_{6}^{2} r_{1}^{4}}{m_{1}} \mathrm{yr}=0.044 \frac{P_{2}^{8 / 3}}{m_{1} m_{6}^{2 / 3}} \mathrm{yr} .
$$

LISA or a similar gravitational wave detector is likely to be able to follow a merger event for at most a few years. Let us assume $t_{m}<10 \mathrm{yr}$. The time scale on which the gravitational wave torque acts on the orbit is given by $t_{g w}=8 t_{m}<100 \mathrm{yr}$. The time scale of the hydrodynamic torque (eq 10) is seen to be longer than $t_{g w}$ by nearly ten orders of magnitude (recall that $\dot{m} \lesssim 0.1$ for an ADAF and is probably $\sim 10^{-2}-10^{-4}$ in normal galactic nuclei). Thus it is clear that the hyrdrodynamic torque is completely negligible.

For a more quantitative consideration, let us compute the number of wave periods prior to the merger of the two objects:

$$
n_{m}=\int_{0}^{t_{m}} \frac{d t}{P}=\frac{8}{5} \frac{t_{m}}{P}
$$

Putting in numerical values,

$$
n_{m}=3.1 \times 10^{5} \frac{m_{6} r_{1}^{5 / 2}}{m_{1}}=2.2 \times 10^{4} \frac{P_{2}^{5 / 3}}{m_{1} m_{6}^{2 / 3}}=1.6 \times 10^{5} \frac{t_{m, y r}^{5 / 8}}{m_{1}^{3 / 8} m_{6}^{1 / 4}},
$$

where $t_{m, y r}=t_{m} / 1 \mathrm{yr}$. With a strong signal and a theoretically computed template of the wave train one expects to follow the phase of the gravitational waves with a time resolution of better than a period. Optimistically, one might be able to resolve down to a tenth of a wave period (Kip Thorne, private communication). The importance of the hydrodynamic drag is then estimated by the quantity

$$
\epsilon \equiv 10 n_{m} \frac{t_{g w}}{t_{h d}}=2.8 \times 10^{-5} \frac{\dot{m} m_{1}^{5 / 8} t_{m, y r}^{13 / 8}}{m_{6}^{5 / 4}} .
$$

If $\epsilon>1$, we expect the hydrodynamic perturbation to have a noticeable effect on the inspiral wave form. For any reasonable choice of the parameters, however, we see that $\epsilon \ll 1$, implying that the hydrodynamic drag has a negligible effect on the observed orbital decay of the compact star.

\section{Discussion}

In this paper we have obtained a very robust result, namely that a compact star orbiting inside an advection-dominated accretion flow around a supermassive black hole experiences a negligible amount of frictional drag from hydrodynamic forces. In deriving this result we made conservative assumptions (e.g. we did not take into account the mitigating effects mentioned at the end of $\S 2$ ) and, where possible, we erred on the side of overestimating the drag (e.g. in our estimate of $I$ in eq 7). Yet we found that the perturbation due to hydrodynamic forces, as measured by the parameter $\epsilon$ (eq 16), is always extremely small. To determine how relevant this result is for gravitational wave experiments, we need to consider several questions.

First, what fraction of galactic nuclei contain SMBHs? Recent work has revealed that the fraction is close to unity. In our local group of galaxies, the three largest galaxies, namely M31, M32 and our own 
Milky Way Galaxy, have dark masses in their nuclei with masses in the range $10^{6.5}-10^{7.5} M_{\odot}$. Outside the local group, observations with the Hubble Space Telescope and with ground-based telescopes have revealed dark massive objects in the nuclei of nearly all galaxies that are accessible to sensitive observations (e.g. Magorrian et al. 1998, Richstone et al. 1998); the masses are in the range $10^{6}-10^{9.5} M_{\odot}$. It is considered highly likely that all these dark masses are SMBHs, though this is yet to be proved conclusively. In the case of our own Galactic nucleus and the nucleus of NGC 4258, the argument for a SMBH is quite compelling (Genzel et al. 1997, Ghez et al. 1998, Miyoshi et al. 1995, Narayan et al. 1998).

Second, what fraction of galactic nuclei are inactive or only weakly active, thus indicating highly subEddington accretion? (We consider a nucleus to be very sub-Eddington if its luminosity is less than a few percent of the Eddington luminosity, $L_{E d d}=10^{46}\left(M_{S M B H} / 10^{8} M_{\odot}\right) \mathrm{erg} \mathrm{s}^{-1}$; when the mass of the SMBH is not known, we take the luminosity limit to be $10^{43} \mathrm{erg} \mathrm{s}^{-1}$, which corresponds to $3 \%$ of $L_{E d d}$ for a $10^{6.5} M_{\odot}$ $\mathrm{SMBH}$.$) The fraction of inactive/weakly active nuclei appears to be close to unity. For instance, there are$ no active galactic nuclei in our local group of galaxies or in the nearby Virgo cluster of galaxies. (M87, the dominant galaxy in Virgo, has what may be considered an active nucleus, but its luminosity is highly sub-Eddington for a $2 \times 10^{9} M_{\odot} \mathrm{SMBH}$, cf. Reynolds et al. 1996.) The number density of quasars averaged over a larger volume of the nearby universe is also extremely low (Krolik 1999). If we include the more numerous Seyferts, which have lower luminosities than quasars but still fall under the definition of active nuclei, the local number density is of order $2-3 \%$ of the number density of all galaxies brighter than $L_{*}$ (Huchra \& Burg 1992). The incidence of nuclear activity increases with increasing redshift, reaching a peak at $z \sim 2-3$. However, out to $z \sim 1$, the redshift range accessible to LISA for stellar inspiral events, the fraction of galaxies with active nuclei is very likely no more than $0.1-0.2$. Thus, over the volume of the universe of interest to us, the majority of galactic nuclei have significantly sub-Eddington accretion.

Third, how strong is the evidence that SMBHs with highly sub-Eddington accretion have ADAFs around them? In the opinion of this author, the evidence is fairly strong. For low mass accretion rates such as we are considering, $\dot{M}<0.1-0.01 \dot{M}_{\text {Edd }}$, only two stable, self-consistent, rotating, accretion flow solutions are known (Chen et al. 1995): the thin disk solution and the ADAF solution. For all low luminosity black holes for which sufficient data are available, whether they be in galactic nuclei or in stellar-mass binaries, some version of the ADAF model appears to explain the observations (Narayan, Yi \& Mahadevan 1995, Narayan, McClintock \& Yi 1996, Reynolds et al. 1996, Narayan, Barret \& McClintock 1997, Di Matteo \& Fabian 1997, Manmoto et al. 1997, Hameury et al. 1998, Narayan et al. 1998, Di Matteo et al. 1999, Quataert et al. 1999). In several objects, the evidence suggests that both an ADAF and a thin disk are present, but such that the ADAF is located close to the black hole, while the thin disk is restricted to radii beyond tens, hundreds or even thousands of $R_{g}$. For the gravitational wave experiments that are the focus of this paper, the compact stars will be in orbits with radii smaller than about $10 R_{g}$. This region of the accretion flow is not in the form of a thin disk in any low luminosity black hole that has been studied so far; the evidence suggests that the accretion flow forms an ADAF in all cases.

Finally, if the accretion is not in the form of an ADAF close to the SMBH, what other form might it take, and could the hydrodynamic drag be significant? This is a difficult question to answer since at present the only solutions we are aware of for low values of $\dot{m}$ are the thin disk and the ADAF. One other formal solution is known, due to Bondi (1952), but it corresponds to the unlikely case of zero angular momentum in the accreting gas. In any case, the hydrodynamic drag force on an orbiting star from a Bondi flow is even less (by a factor of $\alpha$ ) than that from an ADAF.

A point worth making is that if the accretion occurs in a non-ADAF mode, then the mass accretion rate must be a lot less than the values $\dot{M}_{\mathrm{SMBH}} \sim 10^{-2}-10^{-4} \dot{M}_{\mathrm{Edd}}$ that we have assumed in this paper. This 
is because the luminosities of the "normal " nuclei we are considering are extraordinarily low. For instance, the SMBH in the nucleus of our own Galaxy has a luminosity $<10^{-7} L_{\text {Edd }}$ (e.g. Narayan et al. 1998) despite an estimated mass accretion rate of $\sim 10^{-4} \dot{M}_{\text {Edd }}$ (Quataert, Narayan \& Reid 1999, and references therein). The mismatch between the luminosity and the accretion rate is natural with an ADAF since the accretion flow advects the missing energy through the event horizon into the black hole. Indeed, this argument has been used to argue for the presence of an event horizon in this and other black hole systems with ADAFs (see Menou, Quataert \& Narayan 1999 for a review). With a non-ADAF model, the accretion rate would have to be $<10^{-7} \dot{M}_{\text {Edd }}$ in our Galactic nucleus, and comparably small in most other low luminosity nuclei. Even without knowing the details of the flow, it is probably safe to assume that the hydrodynamic drag on an orbiting star in such an ultra-low- $\dot{M}$ accretion flow would be insignificant.

We thus conclude that the majority of inspiral events that LISA might detect will be unaffected by hydrodynamic interactions.

Acknowledgments. It is a pleasure to thank Kip Thorne for instigating this study, for useful discussions, and for persuading the author to publish the results, John Huchra for advice on the statistics of active galactic nuclei, and Eve Ostriker for help in understanding the hydrodynamic drag force. This work was supported in part by grant AST 9820686 from the NSF.

\section{References}

Abramovici, A., et al. 1992, Science, 256, 325

Abramowicz, M., Chen, X., Kato, S., Lasota, J. P., \& Regev, O., 1995, ApJ, 438, L37

Abramowicz, M., Czerny, B., Lasota, J. P., \& Szuszkiewicz, E. 1988, ApJ, 332, 646

Bender, P., et al. 1996, LISA. Laser Interferometer Space Antenna for the detection and observation of gravitational waves, Max-Planck Institut fur Quantenoptik, Report No. MPQ 208, Garching, Germany

Blandford, R. D., \& Begelman, M. C., 1999, MNRAS, 303, L1

Bondi, H. 1952, MNRAS, 112, 195

Chakrabarti, S. K. 1993, ApJ, 411, 610

Chakrabarti, S. K. 1996, Phys. Rev. D53, 2901

Chen, X., Abramowicz, M., \& Lasota, J. P. 1997, ApJ, 476, 61

Chen, X., Abramowicz, M., Lasota, J. P., Narayan, R., \& Yi, I. 1995, ApJ, 443, L61

Cutler, C. 1998, Phys. Rev. D57, 7089

Danzmann, K., et al. 1996, LISA Pre-Phase A Report, Max-Planck Institut fur Quantenoptik, Report No. MPQ 208, Garching, Germany

Di Matteo, T., \& Fabian, A. C. 1997, MNRAS, 286, L50

Di Matteo, T., Quataert, E., Allen, S. W., Narayan, R., \& Fabian, A. C., 1999, ApJ, submitted astro-ph/9905052

Esin, A. A., McClintock, J. E., \& Narayan, R. 1997, ApJ, 489, 867

Folkner, W. M., ed. 1998, Laser-Interferometer Space Antenna, AIP Conf Proc. 456 (New York: AIP Press)

Genzel, R., Eckart, A., Ott, T., \& Eisenhauer, F. 1997, MNRAS, 291, 219

Ghez, A. M., Klein, B. L., Morris, M., \& Becklin, E. E. 1998, ApJ, 509, 678

Hameury, J. M., Lasota, J. P., McClintock, J. E., \& Narayan, R. 1997, ApJ, 489, 234

Hils, D., \& Bender, P. 1995, ApJL, 445, L7

Huchra, J., \& Burg, R. 1992, ApJ, 393, 90

Ichimaru, S. 1977, ApJ, 214, 840

Kato, S., Fukue, J., \& Mineshige, S. 1998, Black-Hole Accretion Disks (Kyoto: Kyoto Univ. Press)

Krolik, J. 1999, Active Galactic Nuclei (Princeton: Princeton Univ.)

Magorrian, J., et al. 1998, AJ, 115, 2285

Manmoto, T., Mineshige, S., \& Kusunose, M. 1997, ApJ, 489, 791

Menou, K., Quataert, E., \& Narayan, R. 1999, in Black Holes, Gravitational Radiation, and the Universe, eds. B.R. Iyer \& B. Bhawal (Dordrecht: Kluwer)

Miyoshi, M., Moran, J., Herrnstein, J., Greenhill, L., Nakai, N., Diamond, P., \& Inoue, M. 1995, Nature, 373, 127

Molteni, D., Gerardi, G., \& Chakrabarti, S. K. 1994, ApJ, 436, 249

Narayan, R., Barret, D., \& McClintock, J. E. 1997, ApJ, 482, 448 
Narayan, R., Kato, S. \& Honma, F. 1997, ApJ, 476, 49

Narayan, R., Mahadevan, R., Grindlay, J. E., Popham, R. G., \& Gammie, C. 1998a, ApJ, 492, 554

Narayan, R., Mahadevan, R., \& Quataert, E. 1998, in Theory of Black Hole Accretion Disks, eds. M. A. Abramowicz, G. Bjornsson, \& J. E. Pringle, p148 (Cambridge Univ. Press)

Narayan, R., McClintock, J. E., \& Yi, I. 1996, ApJ, 457, 821

Narayan, R., \& Yi, I. 1994, ApJ, 428, L13

Narayan, R., \& Yi, I. 1995a, ApJ, 444, 231

Narayan, R., \& Yi, I. 1995b, ApJ, 452, 710

Narayan, R., Yi, I., \& Mahadevan, R. 1995, Nature, 374, 623

Novikov, I. D., \& Thorne, K. S. 1973, in Blackholes, eds. C. DeWitt, \& B. DeWitt, p343 (Gordon \& Breach)

Ostriker, E. C. 1999, ApJ, 513, 252

Quataert, E., Di Matteo, T., Ho, L., \& Narayan, R. 1999, ApJ, submitted

Quataert, E., \& Narayan, R. 1999, ApJ, in press astro-ph/9810136)

Quataert, E., Narayan, R., \& Reid, M. J. 1999, ApJ, 517, L101

Rees, M. J., Phinney, E. S., Begelman, M. C., \& Blandford, R. D. 1982, Nature, 295, 17

Reynolds, C. S., Di Matteo, T., Fabian, A. C., Hwang, U., \& Canizares, C. R. 1996, MNRAS, 283, L111

Richstone, D., et al. 1998, Nature, 395A, 14

Ryan, F. D. 1995, Phys. Rev. D52, 5707

Shakura, N. I., \& Sunyaev, R. A. 1973, A\&A, 24, 337

Shapiro, S. L., \& Teukolsky, S. A. 1983, Black Holes, White Dwarfs, and Neutron Stars (New York: Wiley)

Sigurdsson, S. 1997, Class. Q. Grav., 14, 1425 